\begin{tabular}{|c|l|}
\hline Title & Josephson effect in two-band superconductors \\
\hline Author(s) & Sasaki, A kihiro; Ikegaya, Satoshi; Habe, Tetsuro; Golubov, A lexander A.; A sano, Y asuhiro \\
\hline Citation & $\begin{array}{l}\text { Physical Review B, 101/18), 184501 } \\
\text { https://doi.org/10.1103/PhysRevB.101.184501 }\end{array}$ \\
\hline Issue Date & 2020-05-04 \\
\hline Doc URL & http://hdl.handle.net/2115//8398 \\
\hline Rights & Copyright (2020) by The A merican Physical Society. \\
\hline Type & article \\
\hline File Information & PhysRevB.101.184501.pdf \\
\hline
\end{tabular}

Instructions for use 


\title{
Josephson effect in two-band superconductors
}

\author{
Akihiro Sasaki, ${ }^{1}$ Satoshi Ikegaya, ${ }^{2}$ Tetsuro Habe $\odot,{ }^{1}$ Alexander A. Golubov,${ }^{3,4}$ and Yasuhiro Asano ${ }^{1,5}$ \\ ${ }^{1}$ Department of Applied Physics, Hokkaido University, Sapporo 060-8628, Japan \\ ${ }^{2}$ Max-Planck-Institut für Festkörperforschung, Heisenbergstrasse 1, D-70569 Stuttgart, Germany \\ ${ }^{3}$ National Research University Higher School of Economics, 101000 Moscow, Russia \\ ${ }^{4}$ Faculty of Science and Technology and MESA+ Institute for Nanotechnology, University of Twente, 7500 AE Enschede, The Netherlands \\ ${ }^{5}$ Center of Topological Science and Technology, Hokkaido University, Sapporo 060-8628, Japan
}

(Received 13 December 2019; revised manuscript received 5 March 2020; accepted 3 April 2020; published 4 May 2020)

\begin{abstract}
We study theoretically the Josephson effect between two two-band superconductors respecting time-reversal symmetry, where we assume a spin-singlet $s$-wave pair potential in each conduction band. The superconducting phase at the first band $\varphi_{1}$ and that at the second band $\varphi_{2}$ characterize a two-band superconducting state. We consider a Josephson junction where an insulating barrier separates two such two-band superconductors. By applying the tunnel Hamiltonian description, the Josephson current is calculated in terms of the anomalous Green's function on either side of the junction. We find that the Josephson current consists of three components which depend on three types of phase differences across the junction: the phase difference at the first band $\delta \varphi_{1}$, the phase difference at the second band $\delta \varphi_{2}$, and the difference at the center-of-mass phase $\left(\delta \varphi_{1}+\delta \varphi_{2}\right) / 2$. A Cooper pair generated by the band hybridization carries the last current component. We discuss the relation between the Josephson current calculated in theories and that observed in experiments.
\end{abstract}

DOI: 10.1103/PhysRevB.101.184501

\section{INTRODUCTION}

The Josephson effect is a fundamental property of all superconducting junctions consisting of more than one superconductor [1]. The dissipationless electric current $J$ flows through the junction in the presence of difference in superconducting phases across the junction $\delta \varphi$. The current-phase relationship (CPR) depends sensitively on electric and magnetic properties of a material sandwiched by two superconductors $[2,3]$. When the material is an insulator, it is well established that CPR in a spin-singlet $s$-wave junction is sinusoidal (i.e., $\left.J=J_{0} \sin \delta \varphi\right)$ [4].

The expression of CPR is expected to be complicated when the junction energy depends on more than two superconducting phases. For instance, a superconducting state is characterized by two phases in a two-band superconductor such as $\mathrm{MgB}_{2}[5,6]$ and iron pnictides [7,8]. Namely, the phase of the pair potential in the first band $\varphi_{1}$ and that of the second band $\varphi_{2}$ characterize a two-band superconductivity. Theories [9-11] have suggested that either an $s_{++}$state of $\varphi_{1}=\varphi_{2}$ or an $s_{+-}$state of $\varphi_{1}=\varphi_{2}+\pi$ can be realized in pnictides. When such a two-band superconductor couples to a single-band $s$-wave superconductor through an insulator, the junction energy depends on the phase of the single-band superconductor $\varphi_{s}, \varphi_{1}$, and $\varphi_{2}$. Actually, theoretical studies on such Josephson junctions have shown the complicated energy diagrams as a function of these phases [12-16]. Especially, an $s_{+-}$state frustrates the junction energy and stabilizes a spontaneously time-reversal symmetry breaking state near the junction interface $[12,13]$. More complicated CPR would be expected in a Josephson junction consisting of two pnictide superconductors [17] because four superconducting phases enter the junction energy. At present, this is ann open issue.

In this paper, we discuss the Josephson current between two time-reversal-respecting two-band (two-orbital) superconductors. We assume a spin-singlet $s$-wave order parameter in each conduction band and consider the band hybridization as well as the band asymmetry. The coupling between the two superconductors is described by the tunneling Hamiltonian, which includes the band-diagonal hopping term. The Josephson current is calculated by using the anomalous Green's function on either side of the junction. In addition to the Josephson current in the two conduction bands $J_{1} \sin \left(\delta \varphi_{1}\right)$ and $J_{2} \sin \left(\delta \varphi_{2}\right)$, the band hybridization generates the interband pairing correlations which carry a Josephson current of $J_{12} \sin \left[\left(\delta \varphi_{1}+\delta \varphi_{2}\right) / 2\right]$. Here $\delta \varphi_{\lambda}$ is the phase difference across the junction at the $\lambda$ th band for $\lambda=1-2$. It was shown in the presence of time-reversal symmetry that the phase of the band hybridization $\theta$ locks the two superconducting phases in a superconductor as $2 \theta=\varphi_{1}-\varphi_{2}$ [18]. Thus, $\theta$ is a gauge parameter unique to a two-band superconductor. The Josephson current turns out to depend on two relative phases: $\delta \varphi_{1}$ and $\delta \theta$. The former is tunable in experiments, whereas the latter is not tunable. In this paper, we calculate the Josephson current under two different situations: (i) $\delta \theta$ is an intrinsic parameter of a junction, and (ii) two superconductors minimize the junction energy by adjusting $\delta \theta$ spontaneously. The results under case (i) recover those in the previous paper [12], and the junction breaks time-reversal symmetry. As a consequence, the Josephson current has a $\cos \delta \varphi_{1}$ component in some cases. On the other hand, the results under case (ii) show that a Josephson junction always preserves time-reversal 
symmetry at $\delta \varphi_{1}=0$ and that the current-phase relationship is sinusoidal. We also discuss a role of an odd-frequency Cooper pair [19] in the Josephson effect of two-band superconductors [18,20-22].

This paper is organized as follows. In Sec. II, we describe a time-reversal superconducting state in a two-band superconductor in terms of a microscopic Hamiltonian. The solution of the Gor'kov equation is also presented. In Sec. III, we formulate the Josephson current by using a tunnel Hamiltonian between two superconductors. On the basis of the analytical results, the dependence of the Josephson current on temperature is shown by solving the gap equation numerically. In Sec. V, we discuss the current-phase relationship of the Josephson current. The conclusion is given in Sec. VI. Throughout this paper, we use units of $k_{\mathrm{B}}=c=\hbar=1$, where $k_{\mathrm{B}}$ is the Boltzmann constant and $c$ is the speed of light.

\section{TWO-BAND SUPERCONDUCTOR}

\section{A. Hamiltonian}

The mean-field Hamiltonian for a two-band (two-orbital) superconductor is described by

$$
\begin{gathered}
\mathcal{H}=\int d \boldsymbol{r} \Psi^{\dagger}(\boldsymbol{r}) H \Psi(\boldsymbol{r}), \\
\Psi(\boldsymbol{r})=\left[\psi_{1, \uparrow}(\boldsymbol{r}), \psi_{2, \uparrow}(\boldsymbol{r}), \psi_{1, \downarrow}^{\dagger}(\boldsymbol{r}), \psi_{2, \downarrow}^{\dagger}(\boldsymbol{r})\right]^{\mathrm{T}}, \\
H=\left[\begin{array}{cccc}
\xi_{1}(\boldsymbol{r}) & v e^{i \theta} & \left|\Delta_{1}\right| e^{i \varphi_{1}} & 0 \\
v e^{-i \theta} & \xi_{2}(\boldsymbol{r}) & 0 & \left|\Delta_{2}\right| e^{i \varphi_{2}} \\
\left|\Delta_{1}\right| e^{-i \varphi_{1}} & 0 & -\xi_{1}(\boldsymbol{r}) & -v e^{-i \theta} \\
0 & \left|\Delta_{2}\right| e^{-i \varphi_{2}} & -v e^{i \theta} & -\xi_{2}(\boldsymbol{r})
\end{array}\right], \\
\xi_{1}(\boldsymbol{r})=-\frac{\nabla^{2}}{2 m}-\gamma-\epsilon_{F}, \xi_{2}(\boldsymbol{r})=-\frac{\nabla^{2}}{2 m}+\gamma-\epsilon_{F},
\end{gathered}
$$

where $\psi_{\lambda, \sigma}^{\dagger}(\boldsymbol{r})\left[\psi_{\lambda, \sigma}(\boldsymbol{r})\right]$ is the creation (annihilation) operator of an electron with spin $\sigma(=\uparrow$ or $\downarrow)$ at the $\lambda$ th conduction band, $v e^{i \theta}$ denotes the hybridization between the two bands, and $\mathrm{T}$ means the transpose of a matrix. Details of the derivation of Eq. (3) are given in Appendix A of Ref. [18], and an outline of the derivation is explained briefly as follows. We begin with two atomic orbitals belonging to the same parity such as two $d$ orbitals, two $p$ orbitals, and a pair of $s$ and $d$ orbitals. The $\lambda$ th orbital function in an atom at $\boldsymbol{R}_{i}$ is described as $\phi_{\lambda}\left(\boldsymbol{r}-\boldsymbol{R}_{i}\right)$. In a metal, $\phi_{\lambda}\left(\boldsymbol{r}-\boldsymbol{R}_{i}\right)$ and $\phi_{\lambda}\left(\boldsymbol{r}-\boldsymbol{R}_{j}\right)$ can overlap each other when $\boldsymbol{R}_{i}$ and $\boldsymbol{R}_{j}$ point two neighboring atoms as

$$
t_{\lambda}=\int d \boldsymbol{r} \phi_{\lambda}^{*}\left(\boldsymbol{r}-\boldsymbol{R}_{i}\right)\left(\frac{-\nabla^{2}}{2 m}\right) \phi_{\lambda}\left(\boldsymbol{r}-\boldsymbol{R}_{j}\right) .
$$

The kinetic energy of an electron at the $\lambda$ th band given by $\xi_{\lambda}(\boldsymbol{r})$ is a result of such nearest-neighbor hopping, where $\gamma$ describes asymmetry in the two bands. In this paper, we assume that two such conduction bands overlap at the Fermi level. At an atom at $\boldsymbol{R}_{i}$, two orbital functions hybridize each other as

$$
\int d \boldsymbol{r} \phi_{1}^{*}\left(\boldsymbol{r}-\boldsymbol{R}_{i}\right) v_{c}(\boldsymbol{r}) \phi_{\lambda}\left(\boldsymbol{r}-\boldsymbol{R}_{i}\right)=v e^{i \theta},
$$

where $v_{c}$ is the periodic potential due to the lattice structure in real space. Since the function $v_{c}(\boldsymbol{r})$ is not spherically symmetric in a metal, the hybridization of the two orbital functions remains a finite value of $v e^{i \theta}$. Generally speaking, the orbital function is a complex value. The phase of the hybridization $e^{i \theta}$ originates from the difference in phase of the atomic orbital functions. The normal-state Hamiltonian of such a metal is described as

$$
\begin{gathered}
\mathcal{H}_{\mathrm{N}}=\sum_{\alpha} \int d \boldsymbol{r}\left[\psi_{1, \alpha}^{\dagger}(\boldsymbol{r}), \psi_{2, \alpha}^{\dagger}(\boldsymbol{r})\right] H_{\mathrm{N}}\left[\begin{array}{l}
\psi_{1, \alpha}(\boldsymbol{r}) \\
\psi_{2, \alpha}(\boldsymbol{r})
\end{array}\right], \\
H_{\mathrm{N}}=\left[\begin{array}{cc}
\xi_{1}(\boldsymbol{r}) & v e^{i \theta} \\
v e^{-i \theta} & \xi_{2}(\boldsymbol{r})
\end{array}\right],
\end{gathered}
$$

where $\alpha=\uparrow, \downarrow$ indicates the spin of an electron. The phase of hybridization does not play any role in the normal state because the phase shift $\psi_{2} \rightarrow e^{-i \theta} \psi_{2}$ eliminates the phase and the global phase shift of the operator does not change the physics.

We assume a uniform spin-singlet $s$-wave pair potential for each conduction band which is defined by

$$
\Delta_{\lambda}=g_{\lambda}\left\langle\psi_{\lambda, \uparrow}(\boldsymbol{r}) \psi_{\lambda, \downarrow}(\boldsymbol{r})\right\rangle,
$$

where $g_{\lambda}>0$ represents the attractive interaction between two electrons on the Fermi surface of the $\lambda$ th band. In this paper, we do not consider any spin-dependent potentials. Therefore, the Hamiltonian In Eq. (3) is represented in $4 \times 4$ matrix form by extracting the spin $\uparrow$ sector from the particle space and the spin $\downarrow$ sector from the hole space. Time-reversal symmetry of such a Bogoliubov-de Gennes Hamiltonian $H$ is represented by

$$
\mathcal{T} H \mathcal{T}^{-1}=H, \quad \mathcal{T}=\hat{\rho}_{0} \hat{\tau}_{0} \mathcal{K},
$$

where $\mathcal{K}$ means the complex conjugation and $\hat{\rho}_{0}$ and $\hat{\tau}_{0}$ are the unit matrices in two-band space and particle-hole space, respectively. It is clear that Eq. (3) does not satisfy Eq. (10) because of three phase factors, $e^{i \varphi_{1}}, e^{i \varphi_{2}}$, and $e^{i \theta}$. The two superconducting phases in the Hamiltonian can be factorized by the global phase shift of operators as

$$
\begin{gathered}
H=U H^{\prime} U^{*}, \\
U=\operatorname{diag}\left[e^{i \varphi_{1} / 2}, e^{i \varphi_{2} / 2}, e^{-i \varphi_{1} / 2}, e^{-i \varphi_{2} / 2}\right] .
\end{gathered}
$$

When all the elements of $H^{\prime}$ are real numbers, $H$ preserves time-reversal symmetry. Actually, it is possible to show the relation

$$
\mathcal{T}_{U} H \mathcal{T}_{U}^{-1}=H, \quad \mathcal{T}_{U}=U^{2} \mathcal{K},
$$

where $\mathcal{T}_{U}$ is a gauge-dependent time-reversal operator. Under the global phase shift in Eq. (12), however, the phase of the hybridization is transformed as

$$
\theta \rightarrow \theta-\frac{\varphi_{1}}{2}+\frac{\varphi_{2}}{2} \text {. }
$$

Therefore, we conclude that Eq. (3) preserves time-reversal symmetry only when

$$
2 \theta-\varphi_{1}+\varphi_{2}=2 \pi n
$$

is satisfied [18]. The phases of the two pair potentials and that of the hybridization are linked to one another when the superconductor preserves time-reversal symmetry. 
Even in the absence of hybridization, the standard meanfield theory in the two-band system contains interaction terms between $\Delta_{1}$ and $\Delta_{2}$ [23] as

$$
\begin{aligned}
& \mathrm{g}_{12}^{*}\left\langle\psi_{1, \uparrow}(\boldsymbol{r}) \psi_{1, \downarrow}(\boldsymbol{r})\right\rangle \psi_{2, \downarrow}^{\dagger}(\boldsymbol{r}) \psi_{2, \uparrow}^{\dagger}(\boldsymbol{r}) \\
& \quad+\mathrm{g}_{12}\left\langle\psi_{2, \uparrow}(\boldsymbol{r}) \psi_{2, \downarrow}(\boldsymbol{r})\right\rangle \psi_{1, \downarrow}^{\dagger}(\boldsymbol{r}) \psi_{1, \uparrow}^{\dagger}(\boldsymbol{r})+\text { H.c. },
\end{aligned}
$$

where the phase of the interaction potential $g_{12}=\left|g_{12}\right| e^{2 i \theta}$ relates $\varphi_{1}$ and $\varphi_{2}$ [18]. The pair interaction term affects the Josephson current only through the amplitude of the pair potentials as

$$
\Delta_{1} \rightarrow \Delta_{1}+\frac{g_{12}}{g_{2}} \Delta_{2}, \quad \Delta_{2} \rightarrow \Delta_{2}+\frac{g_{12}^{*}}{g_{1}} \Delta_{1} .
$$

We do not consider these interaction terms because they do not change the main conclusions in this paper.

\section{B. Gor'kov equation}

The Gor'kov equation in the Matsubara representation reads

$$
\left[i \omega_{n}-H(\boldsymbol{k})\right]\left[\begin{array}{cc}
\hat{\mathcal{G}} & \hat{\mathcal{F}} \\
\underline{\hat{\mathcal{F}}} & -\underline{\mathcal{G}}
\end{array}\right]_{\left(\boldsymbol{k}, i \omega_{n}\right)}=\check{1},
$$

where $\omega_{n}=(2 n+1) \pi T$ is the fermionic Matsubara frequency, with $T$ being temperature. The particle-hole symmetry implies the relations

$$
\begin{aligned}
& \underline{\hat{\mathcal{G}}}\left(\boldsymbol{k}, i \omega_{n}\right)=\hat{\mathcal{G}}^{*}\left(-\boldsymbol{k}, i \omega_{n}\right), \\
& \underline{\hat{\mathcal{F}}}\left(\boldsymbol{k}, i \omega_{n}\right)=\hat{\mathcal{F}}^{*}\left(-\boldsymbol{k}, i \omega_{n}\right),
\end{aligned}
$$

where $\hat{x}$ denotes a $2 \times 2$ matrix in two-band space. The anomalous Green's function is represented as

$$
\begin{gathered}
\hat{\mathcal{F}}\left(\boldsymbol{k}, \omega_{n}\right)=\frac{1}{Z}\left[\begin{array}{cc}
f_{1} & f_{\mathrm{e}}-i f_{\mathrm{o}} \\
f_{\mathrm{e}}+i f_{\mathrm{o}} & f_{2}
\end{array}\right], \\
Z=Z_{1} Z_{2}+2 v^{2}\left(\omega_{n}^{2}-\xi_{1} \xi_{2}+\left|\Delta_{1}\right|\left|\Delta_{2}\right|\right)+v^{4}, \\
Z_{\lambda}=\xi_{\lambda}^{2}+\left|\Delta_{\lambda}\right|^{2}+\omega_{n}^{2}, \\
f_{1}\left(\boldsymbol{k}, \omega_{n}\right)=-\left[Z_{2}\left|\Delta_{1}\right|+v^{2}\left|\Delta_{2}\right| e^{i 2 n \pi}\right] e^{i \varphi_{1}}, \\
f_{2}\left(\boldsymbol{k}, \omega_{n}\right)=-\left[Z_{1}\left|\Delta_{2}\right|+v^{2}\left|\Delta_{1}\right| e^{-i 2 n \pi}\right] e^{i \varphi_{2}}, \\
f_{\mathrm{e}}\left(\boldsymbol{k}, \omega_{n}\right)=v\left[\xi_{1}\left|\Delta_{2}\right|+\xi_{2}\left|\Delta_{1}\right|\right] e^{i\left(\varphi_{1}+\varphi_{2}\right) / 2} e^{i n \pi}, \\
f_{\mathrm{o}}\left(\boldsymbol{k}, \omega_{n}\right)=\omega_{n} v\left[\left|\Delta_{1}\right|-\left|\Delta_{2}\right|\right] e^{i\left(\varphi_{1}+\varphi_{2}\right) / 2} e^{i n \pi},
\end{gathered}
$$

where $n \pi$ is derived from the right-hand side of Eq. (15). The dispersion relations in the two bands are described by $\xi_{1}=\xi_{k}-\gamma$ and $\xi_{2}=\xi_{k}+\gamma$, with $\xi_{k}=\boldsymbol{k}^{2} / 2 m-\epsilon_{F}$. The diagonal components $f_{1}$ and $f_{2}$ describing the intraorbital pairing correlations are linked to the pair potentials $\Delta_{1}$ and $\Delta_{2}$, respectively. The off-diagonal pairing components $f_{\mathrm{e}}$ and $f_{\mathrm{o}}$ are induced by the hybridization and describe the interorbital pairing correlations. In particular, $f_{o}$ represents a spin-singlet $s$-wave Cooper pair which is antisymmetric under permutation of the two orbitals. As a consequence, $f_{\mathrm{o}}$ is an odd function of $\omega_{n}$. The correlation function changes sign when two electrons exchange their (imaginary) times. We will discuss the contribution of these components to the Josephson current in Sec. III. When the two orbitals are identical to each other, the relation $\left|\Delta_{1}\right|=\left|\Delta_{2}\right|$ would be expected by setting $\mathrm{g}_{1}=\mathrm{g}_{2}$ and $\gamma=0$. In such a symmetric case, the odd-frequency pairing correlation $f_{\mathrm{o}}$ vanishes. In this paper, we introduce the band asymmetry parameter $\gamma$ to realize $\left|\Delta_{1}\right| \neq\left|\Delta_{2}\right|$ and to make $f_{\mathrm{o}}$ finite. The asymmetry between the two bands can be considered also through the difference in the corresponding masses. In Eqs. (24)-(27), the effects of different band masses are described by the dispersion relations $\xi_{1(2)}(\boldsymbol{k})=\boldsymbol{k}^{2} /\left(2 m_{1(2)}\right)-\epsilon_{F}-(+) \gamma$. Therefore, the band asymmetry due to different masses changes the pairing correlations only quantitatively.

Here we also supply the expression of the normal Green's function:

$$
\begin{gathered}
\hat{\mathcal{G}}\left(\boldsymbol{k}, \omega_{n}\right)=\frac{1}{Z}\left[\begin{array}{cc}
g_{1} & g_{12} \\
g_{21} & g_{2}
\end{array}\right], \\
g_{1}\left(\boldsymbol{k}, \omega_{n}\right)=-\left[\left(i \omega_{n}+\xi_{1}\right) Z_{2}+\left(i \omega_{n}-\xi_{2}\right) v^{2}\right], \\
g_{2}\left(\boldsymbol{k}, \omega_{n}\right)=-\left[\left(i \omega_{n}+\xi_{2}\right) Z_{1}+\left(i \omega_{n}-\xi_{1}\right) v^{2}\right], \\
g_{12}\left(\boldsymbol{k}, \omega_{n}\right)=A v e^{i \theta}, \\
g_{21}\left(\boldsymbol{k}, \omega_{n}\right)=A v e^{-i \theta}, \\
A=\left(i \omega+\xi_{1}\right)\left(i \omega+\xi_{2}\right)-v^{2}-\left|\Delta_{1}\right|\left|\Delta_{2}\right| .
\end{gathered}
$$

The gap equations are represented as

$$
\Delta_{\lambda}=-\mathrm{g}_{\lambda} T \sum_{\omega_{n}} \frac{1}{V_{\mathrm{vol}}} \sum_{\boldsymbol{k}} f_{\lambda}\left(\boldsymbol{k}, \omega_{n}\right)
$$

and result in

$$
\begin{aligned}
& \Delta_{1}=T \sum_{\omega_{n}} \frac{1}{V_{\mathrm{vol}}} \sum_{k} \frac{g_{1}}{Z}\left[Z_{2}\left|\Delta_{1}\right|+v^{2}\left|\Delta_{2}\right|\right] e^{i \varphi_{1}}, \\
& \Delta_{2}=T \sum_{\omega_{n}} \frac{1}{V_{\mathrm{vol}}} \sum_{k} \frac{g_{2}}{Z}\left[Z_{1}\left|\Delta_{2}\right|+v^{2}\left|\Delta_{1}\right|\right] e^{i \varphi_{2}} .
\end{aligned}
$$

Generally speaking, in a two-band superconductor, we determine both the amplitude and the phase of the two pair potentials in a self-consistent way by solving the gap equation. In Eqs. (35) and (36), however, the phases of the pair potentials have already been settled self-consistently under the condition in Eq. (15). Thus, our remaining task is to determine $\left|\Delta_{1}\right|$ and $\left|\Delta_{2}\right|$, which is not difficult because of the simple mathematical structure in Eqs. (35) and (36).

\section{AMPLITUDE OF THE JOSEPHSON CURRENT}

\section{A. Current formula}

We discuss the Josephson effect between two superconductors, where the superconducting states of each superconductor are described by Eq. (3). We assume that $\xi_{1}, \xi_{2},\left|\Delta_{1}\right|,\left|\Delta_{2}\right|$, and $v$ are common in the two superconductors. The two 
superconductors are connected by the Hamiltonian

$$
\begin{gathered}
\mathcal{H}_{T}=\sum_{\boldsymbol{k}, \boldsymbol{p}, \sigma}\left[\psi_{L, 1, \boldsymbol{p}, \sigma}^{\dagger}, \psi_{L, 2, \boldsymbol{p}, \sigma}^{\dagger}\right] \hat{t}_{\mathrm{T}}\left[\begin{array}{l}
\psi_{R, 1, \boldsymbol{k}, \sigma} \\
\psi_{R, 2, \boldsymbol{k}, \sigma}
\end{array}\right]+\text { H.c. } \\
\hat{t}_{\mathrm{T}}=\left[\begin{array}{cc}
t_{1} & 0 \\
0 & t_{2}
\end{array}\right]
\end{gathered}
$$

where $L(R)$ indicates the superconductor on the left- (right-) hand side of an insulating barrier and $t_{1}$ and $t_{2}$ denote the tunneling elements through the barrier at the first band and that at the second band, respectively. We assume that $t_{1}$ and $t_{2}$ are real numbers and are independent of the wave numbers of an electron. The total Hamiltonian reads

$$
\mathcal{H}_{\mathrm{JJ}}=\mathcal{H}_{L}+\mathcal{H}_{R}+\mathcal{H}_{T}
$$

where $\mathcal{H}_{L(R)}$ is given by Eq. (2) with $\varphi_{1} \rightarrow \varphi_{1 L(1 R)}, \varphi_{2} \rightarrow$ $\varphi_{2 L(2 R)}$, and $\theta_{1} \rightarrow \theta_{L(R)}$. The Josephson current is represented by [24]

$$
J=e \operatorname{Im} \sum_{\boldsymbol{k}, \boldsymbol{p}, \sigma} T \sum_{\omega_{n}} \operatorname{Tr}\left[\hat{t}_{\mathrm{T}} \underline{\hat{\mathcal{F}}}_{R}\left(\boldsymbol{k}, i \omega_{n}\right) \hat{t}_{\mathrm{T}} \hat{\mathcal{F}}_{L}\left(\boldsymbol{p}, i \omega_{n}\right)\right],
$$

where $\operatorname{Tr}$ means the trace over the two-band degree of freedom. Since time-reversal symmetry is preserved in each superconductor, the phases satisfy

$$
\begin{aligned}
& 2 \theta_{L}-\varphi_{1 L}+\varphi_{2 L}=2 \pi n_{L}, \\
& 2 \theta_{R}-\varphi_{1 R}+\varphi_{2 R}=2 \pi n_{R} .
\end{aligned}
$$

By substituting the anomalous Green's function in Eq. (21) into the formula in Eq. (40), we obtain

$$
\begin{gathered}
J=J_{1} \sin \left(\varphi_{1 L}-\varphi_{1 R}\right)+J_{2} \sin \left(\varphi_{2 L}-\varphi_{2 R}\right) \\
+J_{12} \sin \left[\bar{\varphi}_{L}-\bar{\varphi}_{R}+\left(n_{L}-n_{R}\right) \pi\right], \\
\bar{\varphi}_{L}=\frac{\varphi_{1 L}+\varphi_{2 L}}{2}, \bar{\varphi}_{R}=\frac{\varphi_{1 R}+\varphi_{2 R}}{2}
\end{gathered}
$$

where the amplitudes are given by

$$
\begin{aligned}
J_{1}= & J_{0} \frac{T}{T_{c}} \sum_{\omega_{n}} \frac{t_{1}^{2}}{t_{1}^{2}+t_{2}^{2}}\left[\sum_{k} \frac{Z_{2}\left|\Delta_{1}\right|+v^{2}\left|\Delta_{2}\right|}{\pi N_{0} Z}\right]^{2}, \\
J_{2}= & J_{0} \frac{T}{T_{c}} \sum_{\omega_{n}} \frac{t_{2}^{2}}{t_{1}^{2}+t_{2}^{2}}\left[\sum_{k} \frac{Z_{1}\left|\Delta_{2}\right|+v^{2}\left|\Delta_{1}\right|}{\pi N_{0} Z}\right]^{2}, \\
J_{12}= & J_{0} \frac{T}{T_{c}} \sum_{\omega_{n}} \frac{2 t_{1} t_{2}}{t_{1}^{2}+t_{2}^{2}} v^{2}\left[\left\{\sum_{k} \frac{\xi_{2}\left|\Delta_{1}\right|+\xi_{1}\left|\Delta_{2}\right|}{\pi N_{0} Z}\right\}^{2}\right. \\
& \left.-\left\{\sum_{k} \frac{\omega_{n}\left(\left|\Delta_{1}\right|-\left|\Delta_{2}\right|\right)}{\pi N_{0} Z}\right\}^{2}\right], \\
J_{0}= & \frac{\pi T_{c}}{2 e R_{N}}, \quad G_{\mathrm{N}}=4 \pi e^{2}\left(t_{1}^{2}+t_{2}^{2}\right) N_{0}^{2}=R_{\mathrm{N}}^{-1} .
\end{aligned}
$$

The normal conductance $G_{\mathrm{N}}$ is calculated from the normal Green's function in Eq. (28) at $\Delta_{1}=\Delta_{2}=0$, and $N_{0}$ is the normal density of states per spin at the Fermi level. The first term proportional to $J_{1}$ is a result of the tunneling of a Cooper pair between the first conduction band of the two superconductors. In a similar way, the second term represents the Josephson current between the second bands. The third
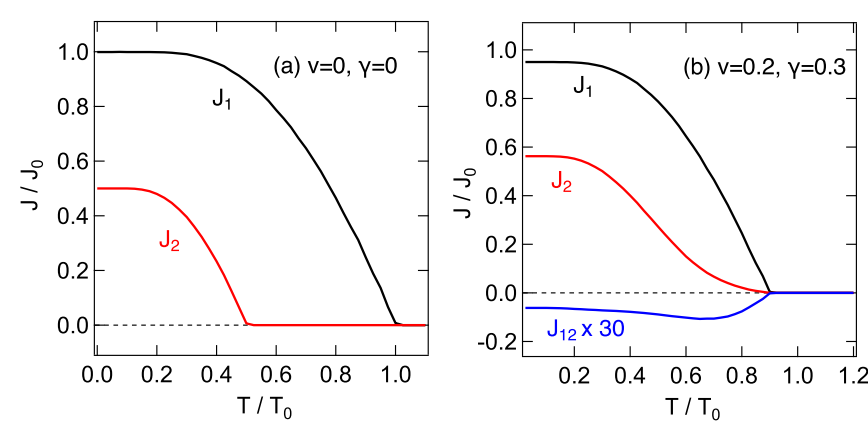

FIG. 1. The dependence of $J_{1}, J_{2}$, and $J_{12}$ on temperature for $\mathrm{g}_{2}=0.5 \mathrm{~g}_{1}$ and $t_{1}=t_{2}=\epsilon_{F}$. The parameters are fixed at $\gamma=v=0$ in (a) and $\gamma=0.2 \epsilon_{F}$ and $v=0.3 \epsilon_{F}$ in (b).

term proportional to $J_{12}$ is the tunneling of an induced Cooper pair by the band hybridization. The hybridization generates two pairing correlations: $f_{e}$ and $f_{o}$ in Eq. (21). The component $f_{e}$ belongs to an even-frequency symmetry class, as shown in Eq. (21), and gives the first term in Eq. (47). On the other hand, the component $f_{o}$ belongs to an odd-frequency symmetry class, as shown in Eq. (21) [21], and gives the second term in Eq. (47). As a result, $J_{12}$ can be negative when the amplitude of $f_{o}$ is larger than that of $f_{e}$. It is known that an odd-frequency Cooper pair indicates the paramagnetic response to magnetic field [21,25,26] and favors the spatial gradient in the phase.

\section{B. Dependence of $\boldsymbol{J}$ on temperature}

The amplitudes of the current in Eqs. (45)-(47) are plotted as a function of temperature in Fig. 1, where we choose $\mathrm{g}_{2}=0.5 \mathrm{~g}_{1}$ and $t_{1}=t_{2}$. The pair potentials $\Delta_{1}$ and $\Delta_{2}$ are calculated by solving the gap equations in Eqs. (35) and (36). The transition temperature at the first band for $v=\gamma=0$ is indicated by $T_{0}$. In Fig. 1(a), $J_{1}$ and $J_{2}$ are shown for $v=\gamma=0$. The two current components are independent of each other, and $J_{12}=0$ in the absence of hybridization. The transition temperature at the second band is about $0.5 T_{0}$ as a result of $g_{2}=0.5 g_{1}$. In Fig. 1(b), we show the results of $J_{1}, J_{2}$, and $J_{12}$ for $v / 2 \pi T_{0}=0.2$ and $\gamma / 2 \pi T_{0}=0.3$. The transition temperature in this case is about $0.9 T_{0}$. All current components are finite at temperatures below $0.9 T_{0}$. The results of $J_{12}$ are amplified by 30 for better visibility and are negative at this parameter choice.

\section{CURRENT-PHASE RELATIONSHIP}

Three phases, $\varphi_{1 j}, \varphi_{2 j}$, and $\theta_{j}$, characterize a two-band superconducting state for $j=L$ and $R$. The condition derived from time-reversal symmetry in Eqs. (41) and (42) reduces the degree of freedom in phases to two. The phase difference $\delta \theta=\theta_{L}-\theta_{R}$ is a parameter characterizing a junction because $\theta_{L(R)}$ is an intrinsic gauge parameter unique to a superconductor. Since $J_{1}>J_{2}$ in Fig. 1, we define the phase across the junction in terms of $\varphi_{1 L(R)}$ by (Fig. 2)

$$
\delta \varphi \equiv \varphi_{1 L}-\varphi_{1 R} .
$$




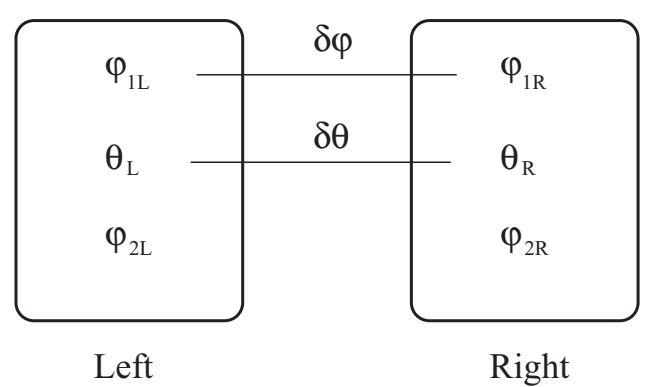

FIG. 2. In a superconductor, the phase difference between the two pair potentials $\varphi_{1 j}-\varphi_{2 j}$ is related to $\theta_{j}$ in Eqs. (41) and (42), which hold true in the presence of time-reversal symmetry. The Josephson current is represented as a function of $\delta \varphi$ and $\delta \theta$.

The phase difference in the second band is described by

$$
\varphi_{2 L}-\varphi_{2 R}=\delta \varphi-2 \delta \theta .
$$

The integer numbers $n_{L}$ and $n_{R}$ in Eqs. (41) and (42) are embedded into $\theta_{L}$ and $\theta_{R}$, respectively. The Josephson current becomes

$$
J=J_{1} \sin \delta \varphi+J_{2} \sin (\delta \varphi-2 \delta \theta)+J_{12} \sin (\delta \varphi-\delta \theta) .
$$

The $\delta \varphi$ corresponds to the phase difference across the junction and is tunable in experiments. On the other hand, $\delta \theta$ is a gauge parameter that depends on a sample. In what follows, we discuss characteristic properties of the current-phase relationship under two different situations: (i) $\delta \theta$ is an intrinsic parameter of a junction, and (ii) $\delta \theta$ is automatically adjusted to minimize the junction energy. When an external (bosonic) degree of freedom couples to $\theta$, the value of $\theta$ can be a sample-specific parameter fixed by such an interaction. When the Josephson coupling energy $E_{J J}$ is much smaller than the interaction energy, $\theta$ could be a static parameter, as considered in case (i). On the other hand, when the Josephson coupling energy is much larger than the interaction, $\theta$ in the two superconductors would be determined self-consistently to minimize the energy of the junction, as discussed in case (ii).

\section{A. Fixed $\delta \theta$}

We discuss typical Josephson junctions by fixing $\delta \theta$ at several values.

(1) $s_{++} / s_{++}$. A two-band superconducting state with $\varphi_{1}=$ $\varphi_{2}$ is called an $s_{++}$state in recent literature and is described by $\theta=0$ or $\pi$ in this paper. We first consider a junction consisting of two $s_{++}$superconductors with $\delta \theta=0$. The resulting current given by

$$
J=\left(J_{1}+J_{2}+J_{12}\right) \sin \delta \varphi
$$

is sinusoidal as a function of the phase difference. It is possible to consider another $s_{++} / s_{++}$junction by choosing $\theta_{L}=\pi$ and $\theta_{R}=0$. The current in such a case results in

$$
J=\left(J_{1}+J_{2}-J_{12}\right) \sin \delta \varphi .
$$

Although the CPR is sinusoidal, the last term changes sign. In an isolated superconductor, the gauge parameter $\theta$ does not affect any observable values because it can be removed by a gauge transformation. In a Josephson junction, however, $\delta \theta$ modifies the Josephson current.
(2) $s_{+-} / s_{+-}$. A two-band superconducting state with $\varphi_{1}=$ $\varphi_{2}+\pi$ is called an $s_{+-}$state and is described by $\theta= \pm \pi / 2$. In a junction consisting of two $s_{+-}$superconductors with $\theta_{L}=$ $\theta_{R}=\pi / 2$, the CPR is given by Eq. (52). However, when we choose $\theta_{L}=\pi / 2$ and $\theta_{R}=-\pi / 2$, the CPR becomes Eq. (53). As in case (1), the CPR is sinusoidal in both cases.

(3) $s_{+-} / s_{++}$. Finally, we consider a junction consisting of an $s_{++}$superconductor and an $s_{+-}$superconductor. The results are summarized as

$$
J=\left(J_{1}-J_{2}\right) \sin \delta \varphi \mp J_{12} \cos \delta \varphi
$$

for $\theta_{L}= \pm \pi / 2$ and $\theta_{R}=0$. When we choose $\theta_{L}= \pm \pi / 2$ and $\theta_{R}=\pi$, the CPR becomes

$$
J=\left(J_{1}-J_{2}\right) \sin \delta \varphi \pm J_{12} \cos \delta \varphi .
$$

The sign change of $J_{2}$ indicates the $\pi$ phase difference in the pair potentials in the second band. The most important feature is the appearance of the $\cos \delta \varphi$ term, which suggests the breakdown of time-reversal symmetry even at $\delta \varphi=0$. According to Eq. (51), the $\cos \delta \varphi$ term always appears when $\delta \theta$ is neither zero nor $\pi$. The obtained results recover the conclusion in a previous paper [12].

\section{B. Varied $\delta \theta$}

The Josephson current is related to the junction energy $E_{\mathrm{JJ}}$ by $J(\delta \varphi)=e \partial_{\delta \varphi} E_{\mathrm{JJ}}$. Namely, the Josephson current vanishes at the ground state. From the relation, the junction energy can be described by

$$
\begin{aligned}
e E_{\mathrm{JJ}}=-J_{1} \cos \delta \varphi & -J_{2} \cos (\delta \varphi-2 \delta \theta)-J_{12} \cos (\delta \varphi-\delta \theta) \\
& \geqslant-\left(J_{1}+J_{2}+\left|J_{12}\right|\right)
\end{aligned}
$$

As shown in Eqs. (45)-(47), $J_{1}$ and $J_{2}$ are always positive, but $J_{12}$ can change sign. We first discuss the case of $J_{12}>0$. As shown in Fig. 1, the first term proportional to $J_{1}$ is the most dominant in our model and takes its minimum at $\delta \varphi=0$. The second and third terms, however, take their minima at $\delta \varphi=$ $2 \delta \theta$ and $\delta \varphi=\delta \theta$, respectively. As we discussed in Sec. II, the gauge parameter $\theta$ does not affect the physics of a single superconductor in the presence of time-reversal symmetry because it is possible to remove it by a unitary transformation. In addition, $\theta$ originates from the difference in phases of two atomic orbital functions $[18,23]$. Therefore, it is reasonable to consider that two superconductors would adjust their gauge parameters $\delta \theta$ to minimize the junction energy spontaneously. Indeed, $E_{\mathrm{JJ}}$ is at its absolute minimum at $(\delta \varphi, \delta \theta)=(0,0)$ for $J_{12}>0$.

For $J_{12}<0$, the junction energy $E_{J J}$ takes its minimum at $(\delta \varphi, \delta \theta)=(0, \pi)$. Namely, the sign change of $J_{12}$ is absorbed as a $\pi$ shift of $\delta \theta$. The CPR remains sinusoidal even at such a nontrivial solution because the $\pi$ shift in $\theta$ does not break time-reversal symmetry as shown in Eq. (15). Thus, we conclude that the CPR is sinusoidal as

$$
J=\left(J_{1}+J_{2}+\left|J_{12}\right|\right) \sin \delta \varphi,
$$

when the two superconductors minimize the junction energy spontaneously by adjusting their gauge parameter $\delta \theta$. 


\section{CONCLUSION}

We studied theoretically the Josephson effect in a tunnel junction consisting of two two-band (two-orbital) superconductors, where the spin-singlet $s$-wave pair potential in each conduction band, the hybridization, and the band asymmetry are considered. In a two-band metal, the superconducting state is characterized by three phases: the phase of the pair potential at the first band $\varphi_{1}$, that at the second band $\varphi_{2}$, and the phase of the band hybridization $\theta$. When time-reversal symmetry is preserved in a two-band superconductor, the relation $2 \theta-$ $\varphi_{1}+\varphi_{2}=2 \pi n$ reduces the phase degree of freedom to two (i.e., $\varphi_{1}$ and $\theta$ ).

On the basis of a standard current formula, the Josephson current is calculated by the anomalous Green's functions on either side of the junction. The Josephson current depends not only on the phase difference across the junction $\delta \varphi_{1}=$ $\varphi_{1, L}-\varphi_{1, R}$ but also on the difference of the gauge parameter $\delta \theta=\theta_{1}-\theta_{2}$ in the two superconductors. The current-phase relationship deviates from the sinusoidal function for $\delta \theta \neq 0$. The dependence of the Josephson current on temperature, the behavior of the gauge parameters in real junctions, and effects of odd-frequency Cooper pairs on the Josephson current are also discussed.

\section{ACKNOWLEDGMENTS}

The authors are grateful to R. Citro, C. Noce, and A. Romano for useful discussions. This work was supported by Topological Materials Science (Grants No. JP15H05852 and No. JP15K21717) from the Ministry of Education, Culture, Sports, Science and Technology (MEXT) of Japan, the JSPS Core-to-Core Program (A. Advanced Research Networks), JSPS and the Russian Foundation for Basic Research under Japan-Russia Research Cooperative Program Grant No. 19-52-50026, and EU H2020-WIDESPREAD-05-2017Twinning project SPINTECH under Grant Agreement No. 810144.
[1] B. Josephson, Phys. Lett. 1, 251 (1962).

[2] K. K. Likharev, Rev. Mod. Phys. 51, 101 (1979).

[3] A. A. Golubov, M. Y. Kupriyanov, and E. Il'ichev, Rev. Mod. Phys. 76, 411 (2004).

[4] V. Ambegaokar and A. Baratoff, Phys. Rev. Lett. 10, 486 (1963).

[5] J. Nagamatsu, N. Nakagawa, T. Muranaka, Y. Zenitani, and J. Akimitsu, Nature (London) 410, 63 (2001).

[6] H. J. Choi, D. Roundy, H. Sun, M. L. Cohen, and S. G. Louie, Nature (London) 418, 758 (2002).

[7] Y. Kamihara, T. Watanabe, M. Hirano, and H. Hosono, J. Am. Chem. Soc. 130, 3296 (2008).

[8] H. Hosono and K. Kuroki, Phys. C (Amsterdam, Neth.) 514, 399 (2015).

[9] K. Kuroki, S. Onari, R. Arita, H. Usui, Y. Tanaka, H. Kontani, and H. Aoki, Phys. Rev. Lett. 101, 087004 (2008).

[10] S. Raghu, X.-L. Qi, C.-X. Liu, D. J. Scalapino, and S.-C. Zhang, Phys. Rev. B 77, 220503(R) (2008).

[11] H. Kontani and S. Onari, Phys. Rev. Lett. 104, 157001 (2010).

[12] T. K. Ng and N. Nagaosa, Europhys. Lett. 87, 17003 (2009).

[13] V. Stanev and Z. Tešanović, Phys. Rev. B 81, 134522 (2010).
[14] I. B. Sperstad, J. Linder, and A. Sudbø, Phys. Rev. B 80, 144507 (2009).

[15] Y. Yerin and A. N. Omelyanchouk, Low Temp. Phys. 43, 1013 (2017).

[16] A. Brinkman, A. A. Golubov, and M. Yu. Kupriyanov, Phys. Rev. B 69, 214407 (2004).

[17] C. Nappi, F. Romeo, E. Sarnelli, and R. Citro, Phys. Rev. B 92, 224503 (2015).

[18] Y. Asano and A. A. Golubov, Phys. Rev. B 97, 214508 (2018).

[19] V. L. Berezinskii, JETP Lett. 20, 287 (1974).

[20] A. M. Black-Schaffer and A. V. Balatsky, Phys. Rev. B 88, 104514 (2013).

[21] Y. Asano and A. Sasaki, Phys. Rev. B 92, 224508 (2015).

[22] Y. Asano, A. Sasaki, and A. A. Golubov, New J. Phys. 20, 043020 (2018).

[23] A. J. Leggett, Prog. Theor. Phys. 36, 901 (1966).

[24] G. D. Mahan, Many-Particle Physics (Plenum, New York, 1990).

[25] Y. Asano, A. A. Golubov, Y. V. Fominov, and Y. Tanaka, Phys. Rev. Lett. 107, 087001 (2011).

[26] S.-I. Suzuki and Y. Asano, Phys. Rev. B 89, 184508 (2014). 